\title{
LAS ADMINISTRACIONES PÚBLICAS \\ EN LA ERA DEL GOBIERNO ABIERTO. \\ GOBERNANZA INTELIGENTE PARA UN CAMBIO \\ DE PARADIGMA EN LA GESTIÓN PÚBLICA ${ }^{1}$ \\ Public administration in the open government age. \\ Smart governance for a paradigm shift \\ in public management
}

\author{
J. IGNACIO CRIADO \\ Universidad Autónoma de Madrid
}

Cómo citar/Citation

Criado, J. I. (2016). Las administraciones públicas en la era del gobierno abierto.

Gobernanza inteligente para un cambio de paradigma en la gestión pública.

Revista de Estudios Políticos, 173, 245-275.

doi: http://dx.doi.org/10.18042/cepc/rep.173.07

\section{Resumen}

La gestión de las administraciones públicas se encuentra en proceso de transformación, tal y como otras dimensiones de la política, dentro de un entorno en que se están produciendo importantes cambios en la base tecnológica de nuestras sociedades. Este artículo ofrece una aproximación teórica, así como evidencia empírica acerca de los cambios recientes en la gestión pública contemporánea derivados de la adopción, uso y difusión de las tecnologías sociales dentro de las organizaciones públicas. En concreto, se sostiene la emergencia de un nuevo paradigma en la gestión pública denominado gobernanza inteligente (smart governance), que se compara con otros paradigmas previos en base a unos fundamentos nuevos, sobre todo: valores derivados de la filosofía 2.0, datos abiertos y transparencia, redes sociales digitales, orientación hacia la incorporación de la ciudadanía y el conocimiento externo a las organizaciones en los procesos de adopción de decisiones públicas y nuevas dinámicas de colaboración entre empleados y organizaciones públicas (Criado, 2016; Noveck, 2015). Lo

1 Este artículo se ha beneficiado de la financiación del Programa S2015/HUM-3466 NEW TRUST-CM (Comunidad de Madrid) y del Proyecto CSO2013-48641-C21-R (Ministerio de Economía y Competitividad). 
anterior se analiza empíricamente a través del análisis de cuatro casos, dos plataformas sociales de crowdsourcing ciudadano (Challenge.gov y SantanderCityBrain) y dos plataformas sociales de colaboración abierta entre empleados públicos (GitHub y NovaGob). Las conclusiones de este estudio permiten vislumbrar las últimas transformaciones en la base tecnológica de las administraciones públicas, así como discutir en qué medida incidirán en cómo entendemos el futuro del sector público.

\section{Palabras clave}

Gestión pública; gobernanza; colaboración; innovación pública; gobierno abierto; tecnologías sociales; participación 2.0.

\section{Abstract}

Management of public administrations is in a process of transformation, as also occurs in other political dimensions, within a context of change in the technological base of our societies. This article offers a theoretical approach, as well as empirical evidence, regarding recent moves in contemporary public management derived from the adoption, use, and diffusion of social media technologies in public organizations. This article supports the idea of an emerging paradigm in public management denoted as 'smart governance', which is compared with previous paradigms on the basis of renewed principles including values derived from philosophy 2.0, open data and transparency, social media platforms, a focus on citizen participation and external knowledge in the decision-making process, and new collaboration dynamics among public employees and organizations (Criado, 2016; Noveck, 2015). This paradigm shift is empirically investigated through four case studies, two social media platforms for citizen crowdsourcing (Challenge.gov y SantanderCityBrain) and two social media platforms for open collaboration among public employees (GitHub y NovaGob). The conclusions of the study shed light on the most recent transformations in the technological dimension of public administration, and discusses to what extent they will impact on our way of understanding the future of the public sector.

\section{Keywords}

Public management; governance; collaboration; public innovation; open government; social technologies; participation 2.0. 


\section{SUMARIO}

I. OBERTURA. II. UN NUEVO PARADIGMA EN LA GESTIÓN PÚBLICA: LA GOBERNANZA PÚBLICA INTELIGENTE. III. LA EVOLUCIÓN DE LOS PARADIGMAS EN LA GESTIÓN PÚBLICA Y SU RELACIÓN CON LAS TIC. IV. ESTUDIOS DE CASO SOBRE LAS NUEVAS TECNOLOGÍAS SOCIALES EN LA GESTIÓN PÚBLICA. 1. Plataformas de crowdsourcing ciudadano. Challenge.gov y SantanderCityBrain. 2. Plataformas de colaboración abierta entre empleados públicos. GitHub y NovaGob. V. CONCLUSIÓN. REFERENCIAS.

\section{OBERTURA}

Este artículo se adentra en los cambios que están iniciándose en un buen número de administraciones públicas contemporáneas como consecuencia de la incorporación de la última generación de tecnologías de información y comunicación (TIC), las denominadas tecnologías sociales. Este trabajo sostiene que la gestión pública se halla en un proceso de transformación, tal y como se manifiesta en otros órdenes de la vida económica, social, cultural o política, como resultado del proceso de adaptación a la nueva ola de cambio tecnológico en marcha, cimentada sobre unas nuevas bases de carácter abierto, colaborativo y relacional. A pesar de que no es posible establecer el alcance de los resultados de tales transformaciones, ni sus implicaciones en el desarrollo futuro de las administraciones públicas, este artículo anuncia que ese proceso de cambio en la gestión de las administraciones públicas implica la llegada de un nuevo paradigma, denominado gobernanza pública inteligente. En línea con lo ya apuntado por la literatura internacional, este paradigma todavía emergente se fundamentaría en nuevas capacidades organizativas e implicaciones para la relación entre administraciones públicas y ciudadanía, la existencia de una serie de tecnologías sociales, así como la adopción de unos valores nuevos de funcionamiento en el sector público más horizontales, abiertos al entorno y con una clara orientación a la innovación.

Desde una perspectiva teórica, existe abundante literatura que muestra que la evolución de la gestión de las administraciones públicas ha transitado de la mano de las transformaciones en las sociedades contemporáneas, especialmente, aquellas relacionadas con los cambios tecnológicos. De hecho, diversos autores han mostrado su interés por entender las interacciones entre las TIC y las administraciones públicas para, a su vez, ser capaces de comprender 
sus implicaciones en la gestión pública contemporánea utilizando enfoques propios de las ciencias sociales (Bekkers y Korteland, 2006; Criado et al., 2013; Fountain, 2001; Gil-García, 2012; Hood y Margetts, 2007; Mergel, 2012a). Lo anterior, no sólo con la intención de conocer el potencial de unas u otras herramientas tecnológicas sobre la eficiencia o eficacia del sector público, sino también porque las tecnologías han acompañado durante las últimas décadas a los diferentes paradigmas en la gestión pública (Criado, 2009; Dunleavy et al., 2008). En concreto, conceptos como innovación abierta, apertura de datos, gamificación, redes sociales digitales o crowdsourcing se van integrando en el vocabulario de los especialistas en la gestión pública, por lo que es imprescindible su entendimiento, también para contribuir a desplegar todo su potencial de mejora en las organizaciones del sector.

Concretamente, este artículo explora el alcance del concepto gobernanza pública inteligente (smart governance), desde la hipótesis de que se trata de un nuevo paradigma en la gestión pública. Hasta el momento, diferentes autores han planteado algunos aspectos sectoriales del concepto, tales como la participación (Charalabidis y Loukis, 2012), el gobierno abierto y la creación de valor público (Harrison et al., 2012) o la colaboración interorganizativa (Gil-García, 2012). En este sentido, las aportaciones más recientes de Scholl y Scholl (2014), Gil-García et al. (2014, 2016) y Noveck (2015) ofrecen una visión más comprehensiva del significado de smart governance y, sobre todo, intentan adentrarse en la naturaleza de los nuevos principios, normas, herramientas y prácticas de lo que parece ser una nueva manera de gobernar lo público (o un nuevo paradigma de la gestión pública). Entre ellos, cabe destacar la incorporación de agentes externos a la organización a los procesos de adopción de decisiones, la difusión de dinámicas colaborativas para poner en marcha proyectos en grupos de trabajo distribuidos, la transparencia radical de los procesos de relación con el entorno o la transformación de las dinámicas de intermediación entre ciudadanía e instituciones. En consecuencia, la lógica smart en la gestión pública se encuentra muy estrechamente unida a la existencia de una nueva generación de tecnologías que no solo permiten la concreción de dinámicas de innovación, sino que además llevan insertos una serie de valores de la web 2.0 o los principios de transparencia, participación y colaboración del gobierno abierto.

Metodológicamente, este artículo pretende combinar una aproximación teórica y empírica a la realidad de la gestión de las tecnologías en las administraciones públicas contemporáneas. En un contexto en el que las tecnologías sociales se han abierto camino en nuestras sociedades, las siguientes páginas tratan de conectar la teoría de la gestión pública, a través de una revisión de los principales paradigmas desarrollados durante las últimas décadas, con evidencia que se deriva del análisis exploratorio de cuatro casos: dos plataformas sociales de crowdsourcing ciudadano (Challenge.gov y SantanderCityBrain) y 
dos plataformas sociales para la innovación abierta entre empleados públicos (GitHub y NovaGob). En estos cuatro casos se traslucen algunas de las características diferenciales del paradigma de la gobernanza pública inteligente, aunque a través de esta estrategia investigadora no se pretenden ofrecer generalizaciones, ni una confirmación de la existencia de un nuevo paradigma en la gestión pública. De una manera más modesta, se aspira a una aproximación sistemática a un objeto de estudio que se encuentra en proceso de consolidación, contribuyendo con ello al debate sobre la smart governance, cada vez más presente en la literatura internacional.

El artículo se divide de la siguiente manera, a partir de la presente sección. En la siguiente se ofrece un recorrido por las principales corrientes que han inspirado la gestión pública contemporánea, desde la Administración Pública tradicional, pasando por la nueva gestión pública o la gobernanza pública, hasta lo que denominamos aquí gobernanza inteligente, tratando de evaluar sus dimensiones clave y las aportaciones más relevantes a las administraciones públicas contemporáneas. A continuación, y de cara a entender mejor las nuevas tendencias en la gestión pública, se explica sucintamente cómo se ha producido la intersección entre las TIC y la gestión pública durante las últimas décadas, de cara a reconocer la situación actual en la que una nueva ola de innovación tecnológica estaría transformando las oportunidades de las administraciones públicas, gracias a su coincidencia con unos valores asociados a la colaboración, la filosofía de la web 2.0 o el redescubrimiento del open government (y sus pilares de transparencia, participación y colaboración). A continuación, se presenta el análisis de los cuatro casos mencionados que todavía se encuentran en una fase emergente, pero que ya permiten vislumbrar el alcance y algunos resultados derivados de su implementación. Para concluir, el artículo se completa con unas reflexiones que resumen las principales contribuciones del artículo y abren nuevas líneas de trabajo para el futuro en el ámbito de las relaciones entre administraciones públicas y TIC.

\section{UN NUEVO PARADIGMA EN LA GESTIÓN PÚBLICA: LA GOBERNANZA PÚBLICA INTELIGENTE}

Las últimas cuatro décadas han experimentado un intenso debate en la gestión pública en relación al origen de las reformas administrativas y herramientas aplicadas en el sector público, no solo en el ámbito de la OCDE, sino también en los países emergentes. A partir de finales de los años setenta, el modelo de Administración Pública inspirada en el ideal burocrático weberiano o Administración Pública tradicional (APT), parecía no encajar bien con el desarrollo de los Estados de bienestar y la expansión de las burocracias públicas para 
la prestación de servicios generalistas (educativos, sanitarios, etc.), que iban más allá de la garantía de derechos y libertades, fundamento de las administraciones públicas del siglo xix y principios del xx. Así, al calor ideológico de los cambios en los gobiernos de Thatcher en el Reino Unido y Reagan en los Estados Unidos se empezaron a plantear ideas alternativas. Por un lado, se comenzó a hablar de la necesidad de incorporar instrumentos y lógicas de acción importados del sector privado bajo la rúbrica de la nueva gestión pública (NGP) (Aucoin, 1995; Barzelay, 2001; Boyne, 2002; Christensen y Laegreid, 2007; Hughes, 2012). Desde otras fuentes filosóficas, se comenzó a poner sobre la mesa la pérdida de centralidad del papel del Estado y la necesidad de abrir la gestión de los asuntos públicos a la interacción con el entorno, en base a la idea de gobernanza pública, con una orientación hacia la colaboración de redes de actores para la configuración de las políticas o la prestación de los servicios públicos (Kettl, 2000; Kickert, 1997; Mayntz, 1998; Peters y Pierre, 1998; Rhodes, 1996). En definitiva, dos nuevos paradigmas en la gestión pública que han tenido distinto éxito en su aterrizaje dentro del gobierno de lo público.

Junto con esos dos grandes paradigmas en la gestión pública, en paralelo se ha desencadenado un proceso de revolución tecnológica de enorme calado. En línea con lo que Castells (2001) identificó hace casi dos décadas como era de la información o sociedad red, con el cambio de milenio, la llegada de ese fenómeno a la gestión pública se ha producido a través de conceptos como administración electrónica, gobierno electrónico o gobierno digital (Criado, 2009; Heeks, 2006; Kraemer y King, 2003; Scholl, 2014; Yildiz, 2007). Estos conceptos se centraron inicialmente en resolver problemas internos de eficiencia o eficacia en línea con la NGP (Dunleavy et al., 2001; Gil-García et al., 2010; Homburg y Bekkers, 2005), así como mejorar la relación con otros actores del entorno mediante la creación de portales web y la prestación de servicios electrónicos siguiendo los postulados de la gobernanza pública. Incluso se llegó a acuñar el término digital era governance para presentar ese nuevo enfoque en la gestión pública (Dunleavy et al., 2006). Solo al iniciarse la segunda década del nuevo milenio, las transformaciones ligadas a la nueva generación de tecnologías y medios sociales, la filosofía de la web 2.0, la ética hacker y la revolución de los datos abiertos, así como la generalización de los principios y valores de la transparencia, participación y colaboración, estarían confluyendo para inspirar una manera diferente de entender la gestión pública en torno a un nuevo modelo de Estado abierto y unas administraciones públicas colaborativas. En consecuencia, a la luz de los avances en la investigación y las experiencias en marcha en diferentes contextos se podría afirmar que, al menos desde un punto de vista teórico, estarían poniéndose las bases de un nuevo paradigma denominado gobernanza pública inteligente (GPI), como siguiente fase en la evolución de la gestión pública contemporánea (véase la tabla 1). 


\section{Tabla 1. Principales paradigmas en la gestión pública contemporánea}

\begin{tabular}{|c|c|c|c|c|}
\hline & $\begin{array}{c}\text { Administración } \\
\text { Pública } \\
\text { Tradicional } \\
\end{array}$ & $\begin{array}{l}\text { Nueva Gestión } \\
\text { Pública }\end{array}$ & $\begin{array}{l}\text { Gobernanza } \\
\text { Pública }\end{array}$ & $\begin{array}{l}\text { Gobernanza Pública } \\
\text { Inteligente }\end{array}$ \\
\hline $\begin{array}{l}\text { Filosofía de } \\
\text { referencia }\end{array}$ & $\begin{array}{l}\text { Teoría política } \\
\text { liberal. Modelo } \\
\text { burocrático } \\
\text { weberiano }\end{array}$ & $\begin{array}{l}\text { Teoría económica. } \\
\text { Gerencialismo } \\
\text { (taylorismo) y } \\
\text { nueva economía } \\
\text { institucional }\end{array}$ & $\begin{array}{l}\text { Teoría política } \\
\text { democrática. } \\
\text { Enfoques } \\
\text { contingentes }\end{array}$ & $\begin{array}{l}\text { Teoría política democrática. } \\
\text { Open government. Economía } \\
\text { colaborativa }\end{array}$ \\
\hline Idea fuerza & $\begin{array}{l}\text { Cumplimiento de } \\
\text { normas y reglas }\end{array}$ & $\begin{array}{l}\text { Búsqueda de } \\
\text { la eficiencia } \\
\text { económica }\end{array}$ & $\begin{array}{c}\text { Construcción } \\
\text { de coaliciones } \\
\text { para lograr } \\
\text { acuerdos sobre } \\
\text { las necesidades } \\
\text { sociales } \\
\end{array}$ & $\begin{array}{c}\text { Creación de valor público a } \\
\text { través de la colaboración con } \\
\text { la ciudadanía }\end{array}$ \\
\hline $\begin{array}{l}\text { Tipo de } \\
\text { Estado y } \\
\text { Administración } \\
\text { Pública }\end{array}$ & $\begin{array}{l}\text { Estado garante / } \\
\text { administración } \\
\text { burocrática }\end{array}$ & $\begin{array}{l}\text { Estado regulador } \\
\text { / administración } \\
\text { postburocrática }\end{array}$ & $\begin{array}{l}\text { Estado regulador } \\
\text { / administración } \\
\text { postburocrática }\end{array}$ & $\begin{array}{c}\text { Estado abierto y delgado / } \\
\text { lean state / administración } \\
\text { abierta, transparente y } \\
\text { colaborativa }\end{array}$ \\
\hline $\begin{array}{l}\text { Papel del } \\
\text { gobierno }\end{array}$ & Remar & Timonear & $\begin{array}{c}\text { Timonear / } \\
\text { construir redes }\end{array}$ & $\begin{array}{l}\text { Construir redes / crear valor } \\
\text { público }\end{array}$ \\
\hline $\begin{array}{l}\text { Modelo de } \\
\text { interacción } \\
\text { social }\end{array}$ & Jerarquía & Mercado & Red & Comunidad \\
\hline $\begin{array}{l}\text { Diseño } \\
\text { organizativo }\end{array}$ & $\begin{array}{l}\text { Unidades } \\
\text { administrativas } \\
\text { integradas }\end{array}$ & $\begin{array}{l}\text { Desagregación } \\
\text { de unidades } \\
\text { en agencias } \\
\text { (agencialización) }\end{array}$ & $\begin{array}{l}\text { Administración } \\
\text { pública en red } \\
\text { y partenariados } \\
\text { público-privados }\end{array}$ & $\begin{array}{l}\text { Administración pública en } \\
\text { red y como plataforma / } \\
\text { halocracia }\end{array}$ \\
\hline $\begin{array}{l}\text { Directivos } \\
\text { públicos }\end{array}$ & $\begin{array}{l}\text { Garantes del } \\
\text { cumplimiento de } \\
\text { las normas y el } \\
\text { procedimiento }\end{array}$ & $\begin{array}{l}\text { Emprendedores } \\
\text { para mejorar } \\
\text { los resultados } \\
\text { en términos } \\
\text { de eficiencia } \\
\text { económica } \\
\end{array}$ & $\begin{array}{l}\text { Dinamizadores de } \\
\text { redes de políticas } \\
\text { públicas }\end{array}$ & $\begin{array}{c}\text { Innovadores expertos en } \\
\text { detectar nichos de creación } \\
\text { de valor público y co-creación } \\
\text { con la ciudadanía }\end{array}$ \\
\hline $\begin{array}{l}\text { Instrumentos } \\
\text { para solucionar } \\
\text { los problemas } \\
\text { públicos }\end{array}$ & $\begin{array}{l}\text { Programas } \\
\text { públicos } \\
\text { altamente } \\
\text { regulados }\end{array}$ & $\begin{array}{l}\text { Herramientas } \\
\text { de gestión } \\
\text { empresarial }\end{array}$ & $\begin{array}{l}\text { Redes de actores } \\
\text { prestadores de } \\
\text { servicios públicos }\end{array}$ & $\begin{array}{l}\text { Herramientas de la web } \\
2.0 \text { (apps, redes sociales, } \\
\text { plataformas colaborativas, } \\
\text { etc.), big data y laboratorios de } \\
\text { innovación }\end{array}$ \\
\hline $\begin{array}{l}\text { Papel de la } \\
\text { ciudadanía }\end{array}$ & Usuario & Cliente & Ciudadano-cliente & Ciudadano co-productor \\
\hline Rol de las TIC & $\begin{array}{l}\text { TIC para la } \\
\text { automatización } \\
\text { de tareas }\end{array}$ & $\begin{array}{c}\text { TIC para la } \\
\text { digitalización } \\
\text { y la eficiencia }\end{array}$ & $\begin{array}{c}\text { TIC para mejorar } \\
\text { la relación } \\
\text { con el entorno } \\
\end{array}$ & $\begin{array}{l}\text { TIC para la innovación } \\
\text { pública colaborativa y la } \\
\text { creación de valor público }\end{array}$ \\
\hline
\end{tabular}

Fuente: Elaboración propia en base a ideas de Arenilla (2012), Criado (2009), Hood (1991), Peters y Pierre (1998) y Pollit (2001). 
Los sucesivos paradigmas en la gestión publica caracterizados en la tabla 1 (APT, NGP, gobernanza pública o GPI) como tipos ideales, se fundamentan en la identificación de una serie de dimensiones que han ido penetrando, con diferentes ritmos e intensidades, en distintos contextos y niveles de gobierno, a lo largo de las últimas décadas, sin que ello signifique que se trate de modelos completamente exhaustivos o totalmente excluyentes entre sí. De hecho, no cabe duda de que en los países con las economías más desarrolladas las administraciones públicas han ido adoptando principios, herramientas, instrumentos, etc., de gestión pública procedentes de varios de los tipos ideales identificados, sin que ello impida afirmar, por ejemplo, el alto impacto de la NGP dentro de los países anglosajones durante los años ochenta y noventa del siglo pasado, o el reciente liderazgo de los Estados Unidos en el desarrollo de las bases del modelo de GPI, bajo el mandato presidencial de Obama. En casos como el español, la prevalencia de dinámicas de funcionamiento procedimentalistas basadas en la APT ha provocado que la aceptación de determinados aspectos procedentes de otros paradigmas se haya producido de una manera desigual (dentro de un mismo nivel de gobierno) y parcial (en aquellas administraciones públicas donde se han asumido determinadas prácticas postburocráticas). Por consiguiente, resulta necesario concretar algo más los aspectos definidos en la tabla 1 de cara a conocer el posible alcance de cada uno de los paradigmas definidos.

A lo largo del siglo xx y hasta la década de los años setenta, la APT tuvo su momento de mayor auge. Lo anterior no impide afirmar su vigencia en cierta medida como paradigma de referencia en determinados contextos y su propia evolución hacia lo que se ha venido a denominar nuevo servicio público. El modelo de la APT surge en el momento de florecimiento de la teoría política liberal, bajo el predominio del modelo burocrático weberiano, que cuenta como eje central de interés el cumplimiento estricto de las normas para garantizar la igualdad de trato de los usuarios para, al menos en teoría, evitar cualquier discrecionalidad del poder público y del funcionariado. En consonancia con ello, el tipo de Estado sobre el que se asienta este modelo es netamente garantista en cuanto a su papel de control sobre el cumplimiento del ordenamiento jurídico, lo que lleva a la existencia de un tipo de administración pública altamente burocratizada y apegada al procedimiento. El papel del gobierno acorde con este paradigma se identifica con la metáfora de llevar los remos (de una embarcación), es decir, asumir las funciones básicas de gestión en un momento en que todavía el Estado de bienestar se encuentra en fase emergente. La jerarquía es el sistema de relación social de referencia y las estructuras ministeriales compactas forman parte del diseño organizativo preferido bajo este paradigma. Otros aspectos clave de este modelo de gestión pública se refieren al papel del directivo público como garante de la norma y el 
recto cumplimiento del procedimiento administrativo, así como la consideración de las personas receptoras de los servicios públicos como usuarias cuya relación con las organizaciones públicas es meramente reactiva.

Este modelo mantiene su interés para el sector público, bien porque en algunos casos no se ha llegado realmente a configurar una administración pública burocrática, bien porque han surgido voces que han alentado una actualización del modelo al mundo contemporáneo. En efecto, diversos países, sobre todo en vías de desarrollo, no han sido capaces de asentar burocracias según la lógica weberiana, lo que ha supuesto una vía abierta a la adopción de otros modelos administrativos, sin disponer de los anticuerpos para eludir algunos de sus potenciales problemas, especialmente, la corrupción y la falta de capacidad profesional y directiva (Ramió, 2001). Al mismo tiempo, autores como Denhardt y Denhardt (2000) plantearon la necesidad de refrescar algunos de los postulados de la APT para adaptarlos a la realidad presente bajo el prisma de lo que han denominado nuevo servicio público (NPS). La definición del NSP se realiza para destacar un movimiento basado en la teoría política democrática, el rescate del papel de la ciudadanía frente a su rol como usuario o cliente, así como la vuelta a un papel estratégico del sector público en la sociedad. De hecho, se puede afirmar que el modelo clásico de administración pública weberiano (o neoweberiano en palabras de Pollit y Bouckaert, 2011) sigue sirviendo de inspiración para la función pública y estando de alguna manera presente entre las opciones de análisis de los especialistas en gestión pública.

El segundo de los modelos de gestión pública identificados aquí se refiere a la NGP. Ya se ha mencionado anteriormente el origen de este paradigma en un momento de puesta en cuestión de los Estados de bienestar, no solo desde el punto de vista de su sostenimiento económico, sino también desde la perspectiva de la gestión operativa de los servicios públicos. En consecuencia con ello, la NGP se inscribe en una filosofía economicista, que entronca con el taylorismo de principios de siglo, pero también con la nueva economía institucional, que en los setenta del siglo pasado adquiere un papel predominante en busca de la eficiencia económica como eje vertebrador de la actividad de las organizaciones públicas. Desde este punto de partida, el Estado va adquiriendo entonces un papel regulador, quedando vaciado de funciones, en la medida que se asume que el sector privado es más eficiente en el control de los recursos para la solución de problemas públicos. Desde una perspectiva organizativa, se genera un intento por desagregar las estructuras burocráticas tradicionalmente unitarias (ministerios), en aras de la creación de organizaciones de misión (agencias, organismos autónomos, empresas públicas, etc.) a las que proponer objetivos mensurables y, a la vez, exigibles a sus directivos, de manera que empieza a gozar de éxito el concepto administración 
pública postburocrática. Adicionalmente, este modelo se inspira en la imagen del directivo público emprendedor, tal y como la dibujaron en su día Osborne y Gaebler (1992), así como la noción de ciudadano-cliente, aspectos que incidían en la necesidad de introducir dinámicas empresariales en las administraciones públicas.

La NGP ha inspirado importantes transformaciones en el sector público durante las últimas tres décadas, con resultados desiguales y, por supuesto, generando efectos deseados, pero también no deseados, por sus promotores. En términos generales, la aceptación del paradigma de la NGP en diferentes contextos partió de la base de la necesidad de actualizar unos patrones de funcionamiento del sector público asentados en un modelo de gestión pública que había nacido en un mundo diferente. En especial, países anglosajones como Australia, Nueva Zelanda o Reino Unido, pero también del entorno europeo continental, incluyendo algunos de los países del sur europeo, plantearon procesos planificados de modernización administrativa sobre la base de estos principios (Alba y Navarro, 2011; Bezes y Parrado, 2013; Barzelay y Gallego, 2010; Ongaro, 2008; Pollit y Bouckaert, 2011; Sotiropoulos, 2015). Más recientemente, no han dejado de plantearse críticas a este enfoque, en parte, por su vinculación con el neoliberalismo económico, en parte, por los propios problemas que la aplicación de determinados principios de la NGP ha supuesto en algunos casos y, también, por las propias incoherencias internas del modelo (Pollit y Bouckaert, 2011). Lo anterior ha llevado a la aparición de nuevas ideas unidas a este paradigma. A modo de ejemplo, los términos whole-of-government o joined up governance han incidido en los problemas derivados de la excesiva fragmentación del sector público y la necesidad de revertirlos con estrategias centradas en una perspectiva holística de la acción gubernamental (Christensen y Laegreid, 2007; Humpage, 2005; Ling, 2002; Pollit, 2003). En todo caso, la NGP sigue estando presente en la caja de herramientas que disponen los directivos públicos en su quehacer diario, así como siendo punto de referencia en los estudios sobre gestión pública contemporáneos.

Por su parte, el concepto gobernanza cuenta con un carácter polisémico dada su aplicación a diferentes contextos (como las relaciones internacionales), que no ha impedido a la gobernanza pública (en red) ser considerada uno de los paradigmas desarrollados durante las últimas décadas en la gestión pública. El origen de este concepto coincide aproximadamente en el tiempo con el surgimiento de la NGP y cuenta con algunos elementos comunes derivados de la realidad compartida en la que se inscriben, así como el tipo de Estado y Administración Pública en que se desarrollan (Bovaird, 2005). No obstante, los referentes teóricos son diferentes, ya que la gobernanza pública no se asienta en un visión economicista, sino más bien en una perspectiva que incide en el papel creciente del entorno y en la necesidad de construir redes de colabo- 
ración con actores públicos-privados para solucionar problemas sociales (Fawcett y Daugbjerg, 2012; Osborne, 2006; Provan y Kenis, 2008; Robichau, 2011). Adicionalmente, este modelo pone el acento en el rol de construcción de coaliciones, que debería ser la principal función de las organizaciones del sector público, así como la superación de las ideas tradicionales de control y jerarquía, frente a la influencia y la red. Complementariamente, el papel de los directivos públicos consiste en consolidar esas redes en torno a políticas y servicios públicos concretos, mediante la aplicación de una serie de conductas directivas que inciden en la colaboración interorganizativa, tales como activar, enmarcar, movilizar o facilitar/sintetizar (Longo, 2012). Este paradigma para la gestión pública ha tenido un elevado impacto a la hora de estudiar y comprender las dinámicas de colaboración público-privadas, por ejemplo, a través de partenariados (PPP en sus siglas en inglés) (Bovaird, 2004; Hodge y Greve, 2007), o bien para abrir una nueva era de los estudios sobre redes de actores, tratando de simplificar la complejidad de gestión de determinadas áreas de política pública.

Las ideas y análisis en torno a la gobernanza pública y la gobernanza de las redes no han parado de crecer en los últimos años como consecuencia del incremento de la complejidad en las sociedades contemporáneas. En este sentido, autores como Klijn (2008) o Rhodes (2007) han vuelto a poner de manifiesto que el término gobernanza se ha usado de maneras diversas, si bien lo más común es el intento de mejorar la coordinación entre actores dependientes con el propósito de alcanzar soluciones a problemas sociales de la manera más óptima posible. En otras palabras, la gobernanza en redes ha puesto sobre la mesa la necesidad de llevar la gestión pública más allá de la comprensión de las herramientas internas de las que disponen las administraciones públicas, para situar el foco de atención en la promoción horizontal de las relaciones entre redes de actores para alcanzar metas colectivas. Lo anterior no es baladí, en la medida que pone en valor propuestas contingentes que van más allá del análisis organizativo interno de la gestión pública, incorporando como eje de interés la conformación de alianzas, que cada vez son más necesarias para afrontar problemas sociales complejos, retorcidos o perversos (wicked-problems) (Head y Alford, 2015; van Bueren et al., 2003). Otras aproximaciones recientes ponen el acento en la pluralidad de propuestas que se derivan de la moderna idea de governance en la relación con el gobierno de lo público (Bevir y Rhodes, 2016; Turnbull, 2016). Consecuentemente, esta tradición administrativa es clave, dado que permite introducir en la agenda numerosas cuestiones de relevancia para las administraciones públicas contemporáneas que siguen imperando en las preocupaciones de académicos y profesionales del sector público.

Dentro de la tabla 1, la GPI representa el enfoque más reciente, que todavía se encuentra en fase de consolidación a mediados de la segunda década del 
nuevo siglo, por lo que se trata de una propuesta en construcción. Desde 2010 se ha producido una aceleración de los cambios derivados de la nueva ola de innovación tecnológica que se encuentra en la base de una nueva manera de entender la política, la administración pública y, por tanto, la gestión pública (Criado, 2013; Subirats, 2012). En particular, este modelo se asentaría en unos principios y valores centrados en la filosofía abierta derivada de la llamada web 2.0 (con la Wikipedia como caso emblemático), la ética hacker colaborativa (Castells, 2013; Himanen, 2002), y la economía colaborativa o del bien común (ligada a la idea de procomún, más que a modelos de negocio predadores como Uber), que en el sector público se traduciría en el movimiento internacional en torno al open government y los pilares de la transparencia, participación y colaboración (Chun et al., 2010; Dassen y Cruz, 2012; Hood, 2011; Jaeger y Bertot, 2010). En este sentido, la teoría política democrática se sitúa como eje vertebrador de una nueva manera de entender la gestión pública que hace de la transparencia, la participación y la rendición de cuentas sus pilares básicos. Además, la colaboración con la ciudadanía no se entiende como una excusa o un proceso exógeno, sino como una necesidad real de integración del conocimiento disperso en la sociedad para identificar y solucionar problemas públicos.

Este modelo surgiría en un momento en que ya pocos desean un Estado sobredimensionado e inflexible, sino más bien un sector público proporcionado, a la vez que ágil, pero, sobre todo, inteligente. Lo anterior se traduce en la capacidad de articulación de la acción de otros actores sociales y la capacidad de integrar lo mejor del entorno en base a la imagen de una administración pública como plataforma (Lathrop y Ruma, 2010). Entonces, aquí la creación de valor público no solo significa que los directivos públicos sean capaces de identificar nichos de interés, sino también de establecer espacios de co-creación de servicios públicos con la ciudadanía (Linders, 2012; Sørensen y Torfing, 2012). La disponibilidad de análisis de datos masivos (big data), junto con la existencia de plataformas sociales (a través de las que interactuar en tiempo real), así como la existencia de sensores y herramientas de escucha activa, permiten orientar la gestión pública hacia la generación de inteligencia en los procesos de gobierno (smart governance) (Noveck, 2015). Esta inteligencia se hace colectiva a través de los portales de transparencia, la conversación continua con la ciudadanía, la evaluación constante derivada de la trazabilidad dentro de los entornos digitales y la generalización de las comunidades de aprendizaje y puesta en marcha de innovación social en la solución de problemas públicos.

El paradigma emergente de la GPI se percibe en las inquietudes y casos de interés de los académicos y expertos en la administración pública contemporánea. Estos se orientan a promover procesos de innovación pública abierta y colaborativa para resolver problemas colectivos junto con la ciudadanía (Challenge.org, SantanderCityBrain, FixMyStreet, etc.) o para aumentar las capacidades 
del sector público de cara a superar sus limitaciones organizativas mediante la colaboración entre empleados públicos (GitHub, NovaGob, GovLoop, etc.) (Clark et al., 2013; Criado y Rojas-Martín, 2015; Criado et al., 2013; Mergel, 2015; Mergel y Desouza, 2013). En este sentido, el nuevo rumbo de la gestión pública contemporánea no significa que se dejen de lado las inquietudes de los enfoques anteriores, sino que más bien estarían poniéndose las bases de una nueva manera de entender la gestión pública basada en el concepto smart, que ya había sido aplicado a las ciudades, pero que ahora alcanza al Estado y se concreta también en gobiernos y administraciones públicas (Chourabi et al. 2012; Gil-García, 2012; Gil-García y Aldama-Alda, 2013). Estos se piensan más abiertos y colaborativos, en la medida que el carburante de la innovación pública es su capacidad relacional. El calado y resultados de las transformaciones ligadas a la GPI no es algo que se pueda predecir, dado que todavía se encuentra en construcción. En todo caso, se trata de una nueva manera de entender la gestión pública desde una perspectiva pluralista, que admite la no existencia de soluciones unívocas para los problemas públicos. Al mismo tiempo, se pone en lugar predominante el papel de la apertura de datos públicos, las nuevas tecnologías sociales y la escucha inteligente de lo que sucede en el entorno, permitiendo a las administraciones públicas pensar en nuevas estrategias para gestionar los procesos internos de trabajo y la relación con la ciudadanía. A continuación se expone de manera más específica el papel que cada uno de los enfoques teóricos presentados ha concedido a las TIC.

\section{LA EVOLUCIÓN DE LOS PARADIGMAS EN LA GESTIÓN PÚBLICA Y SU RELACIÓN CON LAS TIC}

Implícitamente, uno de los puntos de partida de este trabajo consiste en superar las reticencias en la relación de la gestión pública con las tecnologías, sobre todo, en base a la eliminación de prejuicios deterministas. Al menos desde el cambio de siglo, las tecnologías de información y comunicación (TIC) e Internet han concitado parte del interés en el estudio académico y la práctica profesional ligados a las administraciones públicas (Yildiz, 2007). Sin embargo, ese interés por la intersección entre las TIC y el sector público no es algo completamente nuevo. Durante décadas las administraciones públicas han ido adoptando tecnologías en diferentes etapas vinculadas a sus nuevas capacidades (Criado, 2009; Ferro et al., 2011; Gil-García y Luna-Reyes, 2008; Jiménez et al., 2011). Como refleja la tabla 2, se pueden identificar, al menos, cuatro olas de innovación tecnológica dentro de las administraciones públicas, considerando el potencial de las TIC en su relación con ellas. Cada una de esas etapas coincide, quizá con ciertos desajustes temporales, con los diferentes 
paradigmas en la gestión pública analizados en la sección anterior. Esto no implica que la emergencia de los diferentes paradigmas en la gestión pública se deba a las oleadas tecnológicas propuestas, sin embargo, a continuación se mostrará que su alcance y resultados, en cierta medida, ha venido acompañada del tipo de tecnologías disponibles en cada momento.

\section{Tabla 2. Evolución de las Tecnologías de la Información y Comunicación en las Administraciones Públicas}

\begin{tabular}{|c|c|c|c|c|}
\hline $\begin{array}{l}\text { Aspectos de } \\
\text { interés }\end{array}$ & $1950-1970$ & $\begin{array}{c}1980 \text {-mediados } \\
1990\end{array}$ & $\begin{array}{l}\text { Mediados } \\
1990-2010\end{array}$ & 2010- \\
\hline $\begin{array}{l}\text { Tipo de } \\
\text { tecnología }\end{array}$ & Mainframes & $\begin{array}{l}\text { Micro- } \\
\text { informática }\end{array}$ & www & $\begin{array}{l}\text { Plataformas } \\
\text { sociales }\end{array}$ \\
\hline Idea fuerza & Automatizar & Informatizar & Digitalizar & Innovar \\
\hline $\begin{array}{l}\text { Tema clave en } \\
\text { la gestión de } \\
\text { los sistemas de } \\
\text { información }\end{array}$ & $\begin{array}{l}\text { Mantener los } \\
\text { sistemas de } \\
\text { información } \\
\text { operativos y } \\
\text { funcionando }\end{array}$ & $\begin{array}{l}\text { Controlar y } \\
\text { coordinar los } \\
\text { sistemas de } \\
\text { información en } \\
\text { el conjunto de } \\
\text { la organización }\end{array}$ & $\begin{array}{c}\text { Adaptar los } \\
\text { sistemas de } \\
\text { información } \\
\text { para alcanzar } \\
\text { las necesidades } \\
\text { de los clientes } \\
\text { organizativos }\end{array}$ & $\begin{array}{c}\text { Involucrar a la } \\
\text { comunidad y } \\
\text { a los clientes } \\
\text { en los procesos } \\
\text { de innovación: } \\
\text { wikigovernment. } \\
\text { Servir como } \\
\text { plataforma }\end{array}$ \\
\hline Papel de las TIC & Suplantar & Apoyar & Cambiar & Transformar \\
\hline \multirow{2}{*}{$\begin{array}{l}\text { Contenido de } \\
\text { los puestos de } \\
\text { trabajo }\end{array}$} & \multirow{2}{*}{$\begin{array}{l}\text { Sustitución de } \\
\text { la mano de obra } \\
\text { por máquinas }\end{array}$} & $\begin{array}{l}\text { Desarrollo de } \\
\text { recursos de } \\
\text { información de } \\
\text { valor añadido }\end{array}$ & \multirow{2}{*}{$\begin{array}{l}\text { Cambio de } \\
\text { maneras de } \\
\text { hacer }\end{array}$} & \multirow{2}{*}{$\begin{array}{c}\text { Puestos de } \\
\text { trabajo fluidos }\end{array}$} \\
\hline & & $\begin{array}{l}\text { Cambio de } \\
\text { maneras de } \\
\text { pensar y } \\
\text { analizar }\end{array}$ & & \\
\hline $\begin{array}{l}\text { Modelos } \\
\text { organizativos } \\
\text { asociados }\end{array}$ & $\begin{array}{l}\text { Jerarquías } \\
\text { organizativas } \\
\text { centralizadas }\end{array}$ & $\begin{array}{l}\text { Retos a las } \\
\text { fronteras } \\
\text { organizativas, } \\
\text { tanto internas } \\
\text { como externas }\end{array}$ & $\begin{array}{c}\text { Creación } \\
\text { de redes } \\
\text { organizativas }\end{array}$ & $\begin{array}{c}\text { Creación } \\
\text { de redes } \\
\text { organizativas. } \\
\text { Halocracia }\end{array}$ \\
\hline $\begin{array}{l}\text { Normas y } \\
\text { culturas }\end{array}$ & $\begin{array}{l}\text { Culturas/ } \\
\text { normas } \\
\text { tradicionales }\end{array}$ & \multicolumn{2}{|c|}{$\begin{array}{l}\text { Nuevas normas estratégicas y } \\
\text { operativas; cambio de cultura }\end{array}$} & $\begin{array}{l}\text { Filosofía web } \\
\text { 2.0, open } \\
\text { government }\end{array}$ \\
\hline
\end{tabular}

Fuente: Elaboración propia a partir de Criado (2009). 
En un primer momento, las tecnologías en el sector público solo contaban con un papel limitado, muy en línea con las dinámicas de la APT, orientadas a la automatización de tareas. Ese esquema era propio de unas administraciones públicas que empezaron a incorporar las tecnologías de información a partir de mediados del siglo pasado, con el objetivo de suplantar determinadas actividades y cálculos realizados por seres humanos (Bellamy y Taylor, 1998). Las principales herramientas eran los llamados mainframes, que permitieron un importante avance en dos aspectos: primero, desarrollaron la capacidad de las máquinas para realizar tareas de procesamiento numérico a gran escala de forma rápida y, en segundo lugar, mejoraron su capacidad para ser programados (Criado, 2009). Por tanto, las tecnologías de información en ese primer momento se convirtieron en el complemento ideal del modelo tradicional de las grandes organizaciones burocráticas y la APT, con valores de la sociedad industrial y con escasa capacidad para ir más allá de suplantar determinadas tareas internas de gestión.

Las dos etapas siguientes coinciden en el tiempo con la aparición y desarrollo de los enfoques de la NGP y la gobernanza pública, de manera que algunos de sus componentes afectan a esos dos paradigmas, si bien de forma diferente. Desde los años ochenta del siglo pasado, la incorporación de los microordenadores dentro de las organizaciones públicas abrió un período que se identifica con la informatización del sector público (Criado, 2009). Esto coincidió con el incremento de la capacidad del hardware, "cerebro físico" de los ordenadores, para realizar cada vez mayor número de tareas y el desarrollo de la industria del software, o parte lógica-no física, mediante la creación de productos más fáciles para el usuario no especializado. Con ello se concretó un rápido desarrollo y difusión de la informática a un nivel micro y se pusieron en tela de juicio las estructuras de gestión y organizativas existentes y, muy especialmente, los procesos de trabajo (Danziger y Kraemer, 1986). Al mismo tiempo, se produjeron una serie de avances que consolidaron la revolución digital, también dentro de las administraciones públicas, a través de la expansión de las telecomunicaciones. A partir de los años noventa del siglo pasado, las innovaciones en la arquitectura informática y en los sistemas de información no pararon de crecer y estuvieron en la base de los desarrollos actuales de las TIC en las organizaciones públicas.

La aparición de la web y la socialización de Internet supusieron un hito clave para el desarrollo de una nueva lógica en la gestión de las organizaciones orientada al cambio en las formas de hacer dentro del sector público. La versión eficientista de la NGP implicó una utilización de esa capacidad tecnológica para orientarse a la optimización de los procesos internos, la reducción de determinadas cargas administrativas y la digitalización de procesos, en una lógica parecida al desarrollo del comercio electrónico en el sector privado. En otras palabras, se incorporaron en el sector público nuevas aplicaciones deri- 
vadas de Internet y las páginas web que, por obra de sus responsables y no por las tecnologías en sí mismas, se centraron en promover una versión economicista del despliegue de las TIC en la gestión pública (Criado, 2009). Desde una perspectiva externa y en línea con la gobernanza pública, también es cierto que las nuevas tecnologías digitales y sistemas de comunicaciones abrieron las puertas del sector público a otros actores, no solo a través de la puesta a disposición de información o servicios públicos en línea, sino también como consecuencia del desarrollo de sistemas de comunicaciones cada vez más sofisticados y la interoperabilidad entre las administraciones públicas (Criado $e t$ al., 2011; Pardo et al., 2012). Con ello, se evidencia que la manera de involucrar a las TIC en dinámicas más o menos centradas en la NGP o la gobernanza pública no reside tanto en las propias tecnologías, sino en los paradigmas dominantes en la gestión pública en cada momento y lugar.

La última fase de innovación tecnológica cuenta con unas características intrínsecas que están poniendo las bases de verdaderas transformaciones en determinados sectores económicos (las industrias culturales, viajes, prensa, etc.), en nuevos modelos organizativos y, parece que también, en la gestión pública. En este último plano, las capacidades disruptivas de las nuevas tecnologías sociales en el sector público derivan, entre otros, de factores como: 1) la incorporación de los agentes externos de la organización a los procesos de adopción de decisiones, 2) las dinámicas colaborativas que se requieren para poner en marcha determinados proyectos en grupos de trabajo distribuidos (halocracia), 3) la transparencia radical de los procesos de relación con el entorno, 4) la transformación de las dinámicas de intermediación, 5) la reducción de los costes de puesta a disposición de la información, 6) así como la evaluación continuada ligada a la trazabilidad de las acciones en esos nuevos espacios cooperativos digitales (Criado et al., 2013; Criado y Rojas-Martín, 2013; Luna-Reyes y Gil-García, 2014; Mergel, 2012; Mergel y Bretschneider, 2013; Picazo-Vela et al., 2012). Si bien la evidencia empírica todavía es débil, los primeros resultados disponibles muestran que los procesos de innovación abierta y colaborativa desarrollados gracias a esa nueva generación tecnológica llevan inoculado un nuevo modus operandi, capaz de alterar unas relaciones de poder jerárquicas por otras basadas en el mérito, así como una gestión pública cerrada, no participativa y fragmentada, por una gestión pública abierta, participativa y relacional.

\section{ESTUDIOS DE CASO SOBRE LAS NUEVAS TECNOLOGÍAS SOCIALES EN LA GESTIÓN PÚBLICA}

La propuesta teórica de este artículo se completa con una aproximación a cuatro casos en los que las nuevas tecnologías sociales se han aplicado en 
torno a la gestión pública con una lógica ligada a los principios, normas, herramientas y prácticas presentes en la GPI, tal y como se ha presentando antes. De entrada, hay que subrayar que la involucración de la ciudadanía en algunas de estas plataformas sociales desarrolladas por el sector público todavía no resulta masiva, lo que apela a los debates clásicos sobre la brecha digital (Criado y Barrero, 2014; Helbig et al., 2009). Sin embargo, lo importante es que se tenga en cuenta que incluso un número limitado de participantes tiene un alto potencial de cambio institucional como consecuencia de la viralidad, inmediatez y compromiso de aquellos. En esta sección se analizan Challenge. gov y SantanderCityBrain, plataformas sociales orientadas a la incorporación de conocimiento disperso en la sociedad para lograr soluciones colectivas a problemas sociales, bajo la modalidad de crowdsourcing público (Mergel, 2015; Noveck, 2015). Al mismo tiempo, se analizan dos plataformas sociales digitales de colaboración entre empleados públicos de diferentes organizaciones públicas, GITHUB y NovaGob, centradas en la generación de innovación abierta superando las barreras burocráticas convencionales (Mergel, 2012; Criado et al., 2016). Si bien los estudios de caso se construyen en base a un enfoque descriptivo, sin pretensión alguna de generalización, sus resultados pretenden centrarse en la identificación de los principios, normas, herramientas y prácticas del nuevo paradigma de la GPI.

\section{PLATAFORMAS DE CROWDSOURCING CIUDADANO. CHALLENGE.GOV Y SANTANDERCITYBRAIN}

Las plataformas de crowdsourcing ciudadano se orientan a la generación de procesos de producción e innovación colectiva involucrando a determinados colectivos sociales o bien al conjunto de una comunidad política, sobre todo, en el ámbito local. En concreto, las administraciones públicas que las promueven cuentan con un papel esencial a la hora de establecer las tareas y objetivos generales dentro de una comunidad de participantes voluntarios no expertos, normalmente ciudadanos que ofrecen parte de su tiempo y conocimiento dentro de ese espacio y contribuyen en las dinámicas de solución de problemas públicos que plantea la propia organización. En concreto, se puede tratar de concursos de ideas que las administraciones públicas premian mediante algún tipo de incentivo (económico, puntos o de otro tipo) o la involucración de la ciudadanía a través de diferentes medios en la solución de problemas públicos mediante la incorporación de conocimiento o saberes del entorno. En otras palabras, se puede promover el compromiso cívico con los asuntos públicos, se busca la creación de valor público a través de la colaboración directa con la ciudadanía o se orienta la actuación de la Administración Pública hacia una mayor transparencia y capacidad de colaboración con el entorno. 
El caso de Challenge.gov es un ejemplo de una experiencia con un recorrido notable dentro de la Administración Pública en los Estados Unidos de América (EUA). Se trata de un caso derivado del Memorandum del Presidente Obama sobre Transparencia y Gobierno Abierto (White House, 2009) y la iniciativa de la General Services Administration (GSA) para crear una plataforma de apoyo a la estrategia de gobierno abierto mediante un instrumento de política pública denominado "concursos y premios» (White House, 2010). Los principios de la nueva plataforma digital se centraron en el establecimiento de mecanismos para lograr la atracción de nuevas ideas desde cualquier lugar para apoyar los retos ligados al cambio tecnológico, así como ayudar a acometer los retos sociales junto con los desafíos científicos, incluyendo un «Innovator's Tool Kit», con una enumeración de buenas prácticas en el uso de premios y concursos dentro del sector público estadounidense, así como motivando el uso de enfoques de innovación abierta (White House, 2012). De esa manera, se puede decir que es un caso íntimamente ligado a la estrategia de gobierno abierto del presidente Obama y coincide con sus pilares de participación ciudadana y colaboración con otros actores del entorno social.

A día de hoy, Challenge.gov se ha convertido en el modelo de plataforma de crowdsourcing ciudadano más importante a nivel internacional. Su lanzamiento se produjo en septiembre de 2010 y se convirtió en la plataforma social dominante de las agencias federales para promover competiciones para la identificación de soluciones a problemas sociales e incorporar el conocimiento existente fuera de las organizaciones públicas (Mergel y Desouza, 2013). En el momento de cerrarse este artículo se habían lanzado más de 640 competiciones, con más de 220 millones de dólares en premios para los ciudadanos y más de 250.000 participantes, no solo de los EUA, sino también de otros países. El funcionamiento concreto consiste en que, bajo el lema "government challenges, your solutions», las agencias gubernamentales (seekers) proponen un problema o pregunta y cualquier individuo (solvers) responde y envía soluciones. Las agencias gubernamentales solo «retribuyen» con un premio a aquellas soluciones que cumplen los criterios establecidos para alcanzar rentabilidad para la organización y se eligen como ganadoras, normalmente a través de votaciones abiertas. En suma, se trata de un espacio en el que las administraciones públicas involucradas buscan soluciones «bajo demanda», que proceden del entorno ante problemáticas públicas que identifican como relevantes para su actividad. En este caso, resulta especialmente clave la capacidad del gobierno de los EUA para utilizar las tecnologías sociales como un medio para la colaboración con la ciudadanía, convirtiéndola en co-productora de soluciones a problemas colectivos a los que se enfrentan las principales agencias públicas estadounidenses (incluyendo, entre otras, a la $\mathrm{Na}$ tional Aeronautics and Space Administration, Food and Drug Administration o U.S. Census Bureau), que han colaborado en este caso de una manera creciente. 
Por su parte, el caso de SantanderCityBrain confirma las oportunidades de las plataformas ciudadanas de crowdsourcing dentro del contexto local. En este caso, se trata de una plataforma social desarrollada por el Ayuntamiento de Santander, dirigida a todas las personas, sean o no vecinos de la ciudad, que deseen compartir propuestas, comentar y votar las ideas y proyectos que compartan otros usuarios, con el fin de colaborar en el desarrollo de la ciudad, en general, y la ejecución de su plan estratégico, en particular (Ayuntamiento de Santander, 2016). En este caso, una propuesta que estuvo inicialmente orientada a centrarse en la estrategia ciudad inteligente (smart city), se ha terminado ampliando de manera que el Ayuntamiento en cuestión ha identificado una oportunidad para desarrollar una colaboración más intensa con la ciudadanía a través de la captación de ideas sobre temas variados. Dicho de otra manera, se trata de un caso en el que también se pretende la creación de valor público a través de la construcción de redes y la incorporación a la organización municipal de conocimiento disperso en la sociedad.

El caso de SantanderCityBrain es un modelo de desarrollo de una plataforma por parte de una administración local que ha alcanzado un nivel elevado de participación, pero también de concreción del modelo de colaboración abierta con la ciudadanía a través de medios digitales. El número total de ideas planteadas ha superado las 1.300 , con más de 2.500 usuarios, entre proponentes de ideas y otro tipo de participantes. Durante 2016 se ha puesto en marcha un concurso de ideas para decidir áreas en las que destinar un millón de euros del presupuesto municipal. Por tanto, se trata también de una iniciativa que se enmarca dentro de la lógica de "concursos y premios", en la que las personas no solo van planteando propuestas a determinados retos (tal y como plantean casos como Decide Madrid) sino que además van adquiriendo puntos a medida que realizan determinadas acciones dentro de la plataforma. Estas acciones pretenden aumentar el compromiso con la participación y son algunas como registrarse, invitar a registrarse a otro ciudadano, compartir una idea, recibir un voto positivo, emitir un voto positivo o poner un comentario en una idea. Este caso se inserta en el concepto de gamificación (o juegos serios), que se trabaja como medio para lograr una mayor participación e involucramiento de la ciudadanía dentro de plataformas sociales de este tipo. Por todo ello, se trata de un caso que también integra los principios, normas, herramientas y prácticas del nuevo paradigma de la GPI.

\section{PLATAFORMAS DE COLABORACIÓN ABIERTA ENTRE EMPLEADOS PÚBLICOS. GITHUB Y NOVAGOB}

Las plataformas de colaboración abierta entre empleados públicos suponen una manera diferente de generación de innovación colaborativa dentro de 
las administraciones públicas. En este caso, la innovación se plantea a través de la interacción de empleados públicos que se encuentran ubicados en organizaciones diferentes y que comparten algún objetivo común o desean desarrollar algún producto/servicio en colaboración con el trabajo de otros. En estos casos, la creación de valor se sitúa en la ruptura de las lógicas tradicionales de trabajo dentro de una organización o el cumplimiento estricto de las normas, de manera que se trata de desarrollar innovación basada en la integración de ideas y aportaciones de profesionales que pertenecen a organizaciones diferentes para solucionar problemáticas compartidas (Levine y Prietula, 2013; Sørensen y Torfing, 2012). Las implicaciones que se pueden derivar de estas experiencias son variadas y, en todo caso, contienen un alto interés de cara a mejorar la gestión pública desde la propia actuación de los empleados públicos, mediante el intraemprendimiento y en comunidades que rompen los silos organizativos. En estos casos, las plataformas sociales se utilizan para superar barreras espaciales y de tiempo, permitiendo a los empleados públicos desarrollar innovación abierta con pares de otras organizaciones diferentes. En estos casos, también hay una asimilación de los principios, normas, herramientas y prácticas del nuevo paradigma de la GPI.

El caso de GitHub se ha convertido en una plataforma de creación colaborativa de software en la que participan activamente cada vez más administraciones públicas y profesionales relacionados con el sector público. Esta comunidad social de programadores se centra en la reutilización del código creado por otras organizaciones previamente, haciendo cambios para su adaptación al contexto propio de aplicación. Tal y como ha apuntado Mergel (2015: 464) en su análisis del caso de las agencias federales de los EUA, la colaboración no solo se produce para «crear partes de código, tales como elementos de páginas web, o plataformas de datos abiertos, los equipos de investigación gubernamentales también comparten sus data sets, y algoritmos, y recientemente agencias seleccionadas han comenzado a co-desarrollar documentos de política pública en formato texto dentro de GitHub». La plataforma ofrece opciones de interacción social, de manera que las organizaciones y usuarios pueden crear un perfil propio, en el que introducir una fotografía, nombre completo, localización, dirección de correo electrónico y organización a la que pertenecen o a las que se vinculan dentro de la plataforma. Adicionalmente, hay una serie de datos que se muestran en el perfil por defecto, tales como el dato de incorporación a GitHub, el número de seguidores, a quiénes se sigue, así como el número de repositorios que se han destacado o la actividad pública. No obstante, no es necesario estar registrado en la plataforma para consultar los proyectos desarrollados dentro de GitHub.

El caso de GitHub demuestra el potencial para la colaboración abierta entre empleados públicos dentro de una plataforma abierta en la que pueden interaccionar con otros profesionales del sector para desarrollar proyectos con- 
juntamente, tanto de software como de otro tipo. Desde su fundación en febrero de 2008, el número de organizaciones públicas que han desarrollado proyectos de software de manera abierta y colaborativa ha crecido de manera exponencial, incluyendo casos como la National Aeronautics and Space Administration, General Services Administration, Department of Veteran Affairs, pero también otros ligados a la reutilización de datos de la plataforma Data.gov, el repositorio normativo de Congress.gov, la plataforma de peticiones de WhiteHouse.gov, o más recientemente, el desarrollo de la plataforma de gobierno abierto del Ayuntamiento de Madrid. Aunque no se dispone de datos específicos sobre los proyectos relacionados específicamente por organizaciones públicas, se considera que una parte no despreciable de sus 14 millones de usuarios en todo el mundo tiene alguna relación con el sector público. Adicionalmente, Mergel (2015) ha descubierto que las agencias públicas no solo utilizan Gi$t H u b$ para fines propios, sino que también colaboran con organizaciones sin fines de lucro (tales como Code for America o Open Knowledge Foundation), y mediante el análisis de redes sociales (ARS) se puede llegar a determinar las razones que llevan a las organizaciones a colaborar entre sí en este tipo de proyectos. En definitiva, GitHub es una plataforma social que ofrece notables oportunidades de colaboración entre organizaciones públicas, logrando con ello ahorros de costes y generando dinámicas cooperativas de alto valor añadido, no solo para el sector público, sino también para la ciudadanía.

El caso de NovaGob ofrece un ejemplo de plataforma social vertical especializada en el sector público en la que interaccionan sus profesionales de una manera abierta y sin ningún tipo de restricción organizativa. Esta red social profesional especializada en el ecosistema de lo público dentro del ámbito de habla hispana (inspirada en el caso de GovLoop en los EUA y Canadá) es una spin-off o EBC de la Universidad Autónoma de Madrid, creada por un grupo de académicos, empleados públicos y profesionales relacionados con las administraciones públicas. Como han puesto de manifiesto Criado, Rojas-Martín y Villodre (2016) y GovLab (2016), NovaGob conecta a miles de empleados públicos en los países hispanohablantes e incluye a profesionales de todos los niveles de gobierno con el propósito de que desarrollen proyectos de manera abierta y sin el control externo de sus organizaciones de pertenencia. A día de hoy, NovaGob se ha convertido en un ejemplo de colaboración abierta entre empleados públicos, es decir, un espacio en el que la interacción entre los actores participantes es persistente, sin el control de las organizaciones de pertenencia de los profesionales, dado que lo importante son las contribuciones e ideas relacionadas con un propósito común de colaboración o un objetivo concreto (Forte y Lampe, 2013; Mergel, 2015). En definitiva, la plataforma social NovaGob es un espacio social de colaboración abierto a cualquier empleado público que cuenta con un elevado potencial de crecimiento en el futuro. 
El caso de NovaGob también ofrece un ejemplo del poder de la colaboración abierta entre empleados públicos, si bien con un foco más amplio en cuanto a las oportunidades de colaboración y los objetivos planteados. Desde la fundación de la red social en octubre de 2013, NovaGob está cerca de alcanzar los 8.000 miembros registrados en la plataforma procedentes de todos los países de habla hispana, con casi 200 grupos de trabajo, 2000 debates, más de 4.500 entradas/posts de blog, 550 eventos sobre administración pública publicados en una agenda colaborativa, 11000 novatuits o 250 páginas wiki creadas. Adicionalmente, los empleados públicos pueden crear un perfil propio, en el que además de sus datos de referencia profesional o una fotografía, pueden incluir sus datos curriculares, mostrándose sus conexiones o sus principales acciones de interacción en las que participan. Como ha destacado en su estudio GovLab (2016), el centro de la interacción se produce en los grupos de trabajo, orientados a temas o propósitos de colaboración concretos (promoción del gobierno abierto, gestión de redes sociales en el sector público, identificación de retos de futuro de la gestión pública directiva, defensa de la igualdad de género, creación de un lenguaje administrativo claro, compartiendo libros sobre administración pública, promoción de una comunidad de transparencia pública, etc.). Recientemente, también se ha realizado un estudio sobre cómo y por qué se desarrolla la colaboración de los profesionales de lo público dentro de NovaGob (mediante análisis de redes sociales) y las dinámicas de poder que se activan (Villodre, 2016).

Lo importante de todo ello es mostrar que la colaboración abierta entre empleados públicos dispone de un espacio de referencia en el que se desarrollan proyectos específicos, que promueven la innovación en el sector público, superando las limitaciones tradicionales (organizativas, legales, temporales o espaciales), de manera que los intraemprendedores públicos pueden conectarse, compartir sus proyectos y enriquecer a, o ser enriquecidos por, una comunidad activa que crece dentro de un espacio digital de generación de conocimiento y desarrollo de la creatividad. En este caso también se han identificado los principios, normas, herramientas y prácticas del paradigma de la GPI y, al mismo tiempo, se ha puesto de relieve el importante potencial de las tecnologías sociales para configurar una nueva manera de entender la función pública, más centrada en la colaboración interorganizativa y la creación de redes de conocimiento.

\section{CONCLUSIÓN}

El presente artículo ha construido una reflexión teórica y ha aportado evidencias sobre las transformaciones en marcha en la gestión pública contemporánea, centrándose para ello en las oportunidades derivadas de la nueva ola 
de innovación tecnológica y su adopción en el sector público. Por un lado, se ha evidenciado que las últimas cuatro décadas han experimentado importantes debates teóricos en relación con las doctrinas administrativas de referencia en la gestión pública, que han caminado desde la administración pública burocrática weberiana hacia otras propuestas como la nueva gestión pública o la gobernanza pública. Desde un punto de vista teórico, este estudio ha asumido que la evidencia de los últimos años permite vislumbrar la llegada de lo que se ha calificado como gobernanza inteligente o smart governance, un nuevo paradigma en la gestión pública basado en la más reciente ola de innovación tecnológica centrada en las herramientas de social media, unida a principios derivados de la filosofía 2.0 y en línea con una clara orientación hacia prácticas como la incorporación de la ciudadanía en los procesos de adopción de decisiones públicas y con nuevas dinámicas de colaboración entre empleados y organizaciones del sector público. Una vez descritas las bases del nuevo modelo de GPI, el artículo ha profundizado en el papel de las tecnologías en los procesos de transformación de la gestión pública durante las últimas décadas, así como en las características de la nueva ola tecnológica en marcha. A continuación, el artículo ha presentado cuatro casos en relación con, por lado, el crowdsourcing ciudadano, analizando las plataformas Challenge.gov y SantanderCityBrain, y por otro, la colaboración abierta entre empleados públicos, analizando las plataformas GitHub y NovaGob. En todos ellos, se ha evidenciado la existencia de nuevos principios, normas, herramientas y prácticas para el funcionamiento del sector público, pasando de modelos jerárquicos, basados en reglas formales y silos organizativos, hacia un esquema de funcionamiento horizontal, con reglas flexibles y comunidades inter organizativas.

Este artículo ha puesto de manifiesto que las transformaciones de nuestras comunidades políticas también afectan de una manera directa a las administraciones públicas. Como sucede en otras dimensiones de la política, tales cambios se encuentran en proceso de consolidación y solo será posible su incorporación en el sector público si existe una ciudadanía preparada e inteligente, que apoye y dirija la construcción de un Estado inteligente (Noveck, 2015; Criado, 2016). Para ello no solo es necesario transparentar la acción pública u ofrecer mejores espacios de participación ciudadana, sino también establecer bases renovadas para un verdadero cambio en las reglas del juego que han operado durante las últimas décadas en la acción de gobiernos y administraciones públicas. Solo de esa manera será posible superar la desconfianza ciudadana en las instituciones, la falta de legitimidad en determinados actores políticos, así como las carencias de los procesos actuales de apertura gubernamental y participación ciudadana, al mismo tiempo que se adaptan las administraciones públicas a un nuevo modelo de sociedad que todavía se encuentra en proceso de construcción. 
Futuros trabajos relacionados con las administraciones públicas serán necesarios para identificar el alcance de las promesas y su concreción en una mejor gestión de las políticas y servicios públicos. Por un lado, es imprescindible conocer cómo se están implementando en el sector público proyectos relacionados con datos abiertos, transparencia administrativa, participación 2.0, redes sociales, innovación o colaboración abierta. Aquí se requiere más evidencia empírica sobre las implicaciones de los proyectos en marcha que retórica superficial de emprendedores de políticas, si bien ello solo será posible con análisis de carácter longitudinal y sostenidos en el tiempo. Por otro lado, será necesario ampliar el foco de los análisis, dado que muchas de las iniciativas que se han puesto en marcha tienen un alcance global, tal es el caso de GitHub. Pero al mismo tiempo, otras experiencias se identifican con situaciones locales, dado que la creación de valor público se logra en la medida en que los proyectos sean capaces de solucionar problemas públicos concretos de una comunidad política específica. En definitiva, nos encontramos ante un momento de transición hacia un mundo cuyas claves solo podemos intuir, pero que no cabe duda parecen desafiar los principios, normas, herramientas y prácticas que se han dado por asentadas durante las últimas décadas en el ámbito de la gestión de las administraciones públicas.

\section{Referencias}

Alba, C. y Navarro, C. (2011). Administrative tradition and reforms in Spain: Adaptation versus innovation. Public Administration, 89 (3), 783-800. Disponible en: http://dx.doi. org/10.1111/j.1467-9299.2010.01886.x.

Arenilla, M. (coord.) (2011). Crisis y Reforma de la Administración Pública. La Coruña: Netbiblo.

Aucoin, P. (1995). The New Public Management. Canada in Comparative Perspective. Montreal: Institute for Research on Public Policy.

Barzelay, M. (2001). The New Public Management. Improving Research and Policy Dialogue. Berkeley: University of California Press.

Barzelay, M. y Gallego, R. (2010). The Comparative Historical Analysis of Public Management Policy Cycles in France, Italy, and Spain: Symposium Introduction. Governance, 23 (2), 209-223. Disponible en: http://dx.doi.org/10.1111/j.1468-0491.2010.01476.x.

Bekkers, V. J. J. M. y Korteland, E. (2006). Governance, ICT and the Innovation Agenda of Public Administration: A Comparison of some European Policy Initiatives. En V. J. J. M. Bekkers, H. van Duivenboden y M. Thaens (eds.). Information and Communication Technology and Public Innovation (pp. 22-50). Amsterdam: IOS Press.

Bellamy, C. y Taylor, J. A. (1998). Governing in the Information Age. Buckingham: Open University Press.

Bevir, M. y Rhodes, R. A. W. (2016). Rethinking Governance. Ruling, Rationales, and Resistance. Abingdon, Oxon: Routledge. 
Bezes, P. y Parrado, S. (2013). Trajectories of administrative reform: Institutions, timing and choices in France and Spain. West European Politics, 36 (1), 22-50. Disponible en: http://dx.doi.org/10.1080/01402382.2013.742735.

Bovaird, T. (2004). Public-Private Partnerships: From Contested Concepts to Prevalent Practice. International Review of Administrative Sciences, 70 (2), 199-215. Disponible en: http://dx.doi.org/10.1177/0020852304044250.

- (2005). Public governance: Balancing stakeholder power in a network society. International Review of Administrative Sciences, 71 (2), 217-228. Disponible en: http://dx.doi. org/10.1177/0020852305053881.

Boyne, G. A. (2002). Researching the New Public Management. En K. McLaughlin, S. P. Osborne y E. Ferlie (eds.). New Public Management. Current Trends and Future Prospects (pp. 324-338). London: Routledge.

Castells, M. (2001). La era de la información. La sociedad red. 2a ed. Madrid: Alianza. (2013). Comunicación y poder. Madrid: Alianza.

Charalabidis, Y. y Loukis, E. (2012). Participative Public Policy Making Through Multiple Social Media Platforms Utilization. International Journal of Electronic Government Research, 8 (3), 78-97. Disponible en: http://dx.doi.org/10.4018/jegr.2012070105.

Chourabi, H. et al. (2012). Understanding Smart Cities: An Integrative Framework, 45th Hawaii International Conference on System Sciences, Hawaii. Disponible en: http:// dx.doi.org/10.1109/hicss.2012.615.

Christensen, T. y Laegreid, P. (eds.) (2007). Transcending New Public Management: The Transformation of Public Sector Reforms. Aldershot: Ashgate.

- (2007). The whole-of-government approach to public sector reform. Public Administration Review, 67 (6), 1059-1066. Disponible en: http://dx.doi. org/10.1111/j.1540-6210.2007.00797.x.

Chun, S. A., Shulman, S., Sandoval, R. y Hovy, E. (2010). Government 2.0. Making Connections between Citizens, Data and Government. Information Polity, 15, 1-9.

Clark, B. J., Brudney, J. y Jang, S. G. (2013). Coproduction of government services and the new information technology: Investigating the distributional biases. Public Administration Review, 73 (5), 687-701. Disponible en: http://dx.doi.org/10.1111/puar.12092.

Criado, J. I. (2009). Entre sueños utópicos y visiones pesimistas. Madrid: Instituto Nacional de Administración Pública.

- (2013). Open Government, Social Media y Sector Público. En R. Cotarelo (ed.). Ciberpolítica. Las Nuevas Formas de Acción Politica (pp. 57-78). Valencia: Tirant lo Blanch.

- (ed.) (2016). Nuevas tendencias en la gestión pública. Madrid: Instituto Nacional de Administración Pública.

Criado, J. I. y Barrero, D. F. (2014). Analyzing the Digital Divide and Electronic Government Demand. An Empirical Research of the Spanish Case. En M. P. Rodríguez-Bolívar (ed.). Measuring e-Government Efficiency. The Opinions of Public Administrators and Other Stakeholders (pp. 213-232). New York: Springer.

Criado, J. I., Gascó, M. y Jiménez, C. E. (2011). Interoperabilidad de gobierno electrónico en Iberoamérica. Estudio comparativo y recomendaciones de futuro. Reforma y Democracia, 50, 77-104. 
Criado, J. I. y Rojas Martín, F. (eds.) (2013). Las redes sociales digitales en la gestión y las politicas públicas. Avances y desafios para un gobierno abierto. Barcelona: Escola d'Administració Pública de Catalunya.

— (eds.) (2015). Casos de éxito en redes sociales de Administraciones públicas. Barcelona: Escola d'Administració Pública de Catalunya.

Criado, J. I., Rojas-Martín, F. y Villodre, J. (2016). Open collaboration among practitioners and public management researchers in the social media era. The case of NovaGob. Ponencia presentada en la 20th Annual Conference International Research Symposium on Public Management, Hong Kong.

Criado, J. I., Sandoval-Almazán, R. y Gil-García, R. (2013). Government Innovation through Social Media. Government Information Quarterly, 30 (4), 320-328. Disponible en: http://dx.doi.org/10.1016/j.giq.2013.10.003.

Danziger, James N. y Kraemer, K. L. (1986). People and Computers. The Impacts of Computing on End Users in Organizations. New York: Columbia University Press.

Dassen, N. y Cruz, J. (eds.) (2012). Open Government and Targeted Transparency. Trends and Challenges for Latin America and the Caribbean. Washington D.C.: Inter-American Development Bank.

Denhardt, R. B. y Denhardt, J. V. (2000). The New Public Service: Serving Rather than Steering. Public Administration Review, 60 (6), 549-559. Disponible en: http://dx.doi. org/10.1111/0033-3352.00117.

Dunleavy, P., Margetts, H., Bastow, S. y Tinkler, J. (2006). Digital Era Governance: IT Corporations, the State and E-government. Oxford: Oxford University Press. Disponible en: http://dx.doi.org/10.1093/acprof:oso/9780199296194.001.0001.

Dunleavy, P., Margetts, H., Bastow, S., Tinkler, J. y Yared, H. (2001). Policy Learning and Public Sector Information Technology. Contractual and E-Government Changes in the UK, Australia and New Zealand. Ponencia presentada en la Annual Conference of the American Political Science Association, San Francisco.

Fawcett, P. y Daugbjerg, C. (2012). Explaining Governance Outcomes: Epistemology, Network Governance and Policy Network Analysis. Political Studies Review, 10 (2), 195-207. Disponible en: http://dx.doi.org/10.1111/j.1478-9302.2012.00257.x.

Ferro, E., Helbig, N. y Gil-Garcia, J. R. (2011). The Role of IT Literacy in Defining Digital Divide Policy Needs. Government Information Quarterly, 28 (1), 3-10. Disponible en: http://dx.doi.org/10.1016/j.giq.2010.05.007.

Forte, A. y Lampe, C. (2013). Defining, Understanding, and Supporting Open Collaboration Lessons from the Literature. American Behavioral Scientist, 57(5), 535-547.

Fountain, J. E. (2001). Building the Virtual State. Information Technology and Institutional Change. Washington D. C.: Brookings Institution Press.

Gil-García, J. R. (2012). Towards a Smart State? Inter-Agency Collaboration, Information Integration and Beyond. Information Polity, 17 (1), 269-280.

Gil-García, J. R. y Aldama-Alda, A. (2013). Making a City Smarter through Information Integration. Angel Network and the Role of Political Leadership. 46th Hawaii International Conference on System Sciences, Hawaii. 
Gil-García, J. R., Arellano-Gault, D. y Luna-Reyes, L. (2010). Gobierno electrónico en México (2000-2006). Una visión desde la nueva gestión pública. 11th Annual International Digital Government Research Conference, Puebla, México.

Gil-García, J. R. y Helbig, N. (2006). Exploring e-Government Benefits and success factors. Encyclopedia of Digital Government, 2, 803-811.

Gil-García, J. R., Helbig, N. y Ojo, A. (2014). Being smart: Emerging technologies and innovation in the public sector. Government Information Quarterly, 31 (S1), 1-8. Disponible en: http://dx.doi.org/10.1016/j.giq.2014.09.001.

Gil-García, J. R. y Luna-Reyes, Luis F. (2008). Una breve introducción al gobierno electrónico: definición, aplicaciones y etapas. Revista de Administración Pública, 43 (2), 49-72.

Gil-García, J. R. y Pardo, J. (2005). E-Government Success Factors: Mapping Practical Tools to Theoretical Foundations. Government Information Quarterly, 22 (2), 187-216. Disponible en: http://dx.doi.org/10.1016/j.giq.2005.02.001.

Gil-García, J. R., Zhang, J. y Puron-Cid, G. (2016). Conceptualizing smartness in government: An integrative and multi-dimensional view. Government Information Quarterly. Disponible en: http://dx.doi.org/10.1016/j.giq.2016.03.002.

GovLab (2016). NovaGob. Creating a Community of Spanish Speaking Public Employees across National and Institutional Borders. GovLab-Smarter state case studies. Disponible en: http://www.thegovlab.org/static/files/smarterstate/novagob.pdf.

Harrison, T. M., Guerrero, S., Burke, G. B., Cook, M., Cresswell, A., Helbig, N., Hrdinova, J. et al. (2012). Open government and e-government: Democratic challenges from a public value perspective. Information Polity, 17 (2), 83-97.

Head, B. W. y Alford, J. (2015). Wicked Problems: Implications for Public Policy and Management. Administration and Society, 47 (6), 711-739. Disponible en: http://dx.doi. org/10.1177/0095399713481601.

Heeks, R. (2006). Implementing and Managing e-Government. An International Text. London: Sage.

Helbig, N., Gil-García, J. R. y Ferro, E. (2009). Understanding the Complexity of Electronic Government: Implications from the Digital Divide Literature. Government Information Quarterly, 26 (1), 89-97. Disponible en: http://dx.doi.org/10.1016/j.giq.2008.05.004.

Himanen, P. (2002). La ética hacker y el espiritu de la sociedad de la información. Barcelona: Destino.

Hodge, G. A. y Greve, C. (2007). Public-private partnerships: An international performance review. Public Administration Review, 67 (3), 545-558. Disponible en: http://dx.doi. org/10.1111/j.1540-6210.2007.00736.x.

Homburg, V. M. F. y Bekkers, V. J. J. M. (2005). E-Government and New Public Management: a Perfect Marriage? En V. J. J. M. Bekkers y V. M. F. Homburg (eds.). The Information Ecology of e-Government (pp. 155-170). Amsterdam: IOS Press.

Hood, C. (1991). A Public Management for All Seasons? Public Administration, 69 (1): 3-19. Disponible en: http://dx.doi.org/10.1111/j.1467-9299.1991.tb00779.x.

(2011). From FOI world to wikileaks world: A new chapter in the transparency story? Governance, 24 (4), 635-638. Disponible en: http://dx.doi.org/10.1111/ j.1468-0491.2011.01546.x. 
Hood, C. y Margetts, H. (2007). The Tools of Government in the Digital Age. Londres: Palgrave.

Hood, C. y Peters, G. (2004). The Middle Aging of New Public Management: into the Age of Paradox? Journal of Public Administration Research and Theory, 14 (3), 267-282. Disponible en: http://dx.doi.org/10.1093/jopart/muh019.

Hughes, O. (2012). Public Management and Administration. 4a ed. London: Palgrave Macmillan.

Humpage, L. (2005). Experimenting With a «Whole of Government» Approach: Indigenous capacity building in New Zealand and Australia. Policy Studies, 26 (1), 47-66. Disponible en: http://dx.doi.org/10.1080/01442870500041744.

Jaeger, P. T. y Bertot, J. C. (2010). Transparency and technological change: Ensuring equal and sustained public access to government information. Government Information Quarterly, 27 (4), 371-376. Disponible en: http://dx.doi.org/10.1016/j.giq.2010.05.003.

Jiménez, C. E., Criado, J. I. y Gascó, M. (2011). Technological e-Government Interoperability. An Analysis of IberoAmerican Countries. IEEE Latin America Transactions, 9 (7), 1112-1117. Disponible en: http://dx.doi.org/10.1109/TLA.2011.6129711.

Kettl, D. F. (2000). The Transformation of Governance: Globalization, Devolution, and the Role of Government. Public Administration Review, 60 (6), 488-497. Disponible en: http://dx.doi.org/10.1111/0033-3352.00112.

Kickert, W. J. (1997). Public Governance in the Netherlands: an Alternative to Anglo-American Managerialism. Public Administration, 75 (4), 731-752. Disponible en: http:// dx.doi.org/10.1111/1467-9299.00084.

Klijn, E. (2008). Governance and Governance Networks in Europe. An Assessment of Ten Years of Research on the Theme. Public Management Review, 10 (4), 505-525. Disponible en: http://dx.doi.org/10.1080/14719030802263954.

Kraemer, K. L. y King, J. L. (2003). Information Technology and Administrative Reform: Will the Time After e-Government Be Different? Ponencia presentada en Heinrich Reinermann Schrift Fest, 29 de septiembre, Post Graduate School of Administration, Speyer.

Lathrop, D. y Ruma, L. (eds.) (2010). Open government. Collaboration, transparency, and participation in practice, Sebastopol, CA: O'Reilly Media.

Levine, S. y Prietula M. J. (2013). Open Collaboration for Innovation: Principles and Performance. Organization Science, 25 (5), 1414-1433. Disponible en: http://dx.doi. org/10.1287/orsc.2013.0872.

Linders, D. (2012). From e-government to we-government: Defining a typology for citizen coproduction in the age of social media. Government Information Quarterly, 29 (4), 446454. Disponible en: http://dx.doi.org/10.1016/j.giq.2012.06.003.

Ling, T. (2002). Delivering joined-up government in the UK: dimensions, issues and problems. Public administration, 80 (4), 615-642. Disponible en: http://dx.doi. org/10.1111/1467-9299.00321.

Longo, F. (2012). Innovación en gobernanza. Entornos colaborativos y liderazgos relacionales'. Ekonomiaz. Revista Vasca de Economia, 80, 46-69.

Luna-Reyes, L. F. y Gil-García, J. R. (2014). Digital Government Transformation and Internet Portals: The Co-Evolution of Institutions, Technology, and Organizations. Govern- 
ment Information Quarterly, 31 (4), 545-555. Disponible en: http://dx.doi.org/10.1016/ j.giq.2014.08.001.

Mayntz, R. (1998). New Challenges to Governance Theory. Jean Monnet Chair Papers, Instituto Universitario Europeo, The Robert Schman Centre, 50.

Mergel, I. (2012a). Social Media in the Public Sector: A guide to participation, collaboration, and transparency in the networked world. San Francisco: John Wiley and Sons.

(2012b). The social media innovation challenge in the public sector. Information Polity, $17,281-292$.

- (2015). Open collaboration in the public sector: The case of social coding on GitHub. Government Information Quarterly, 32 (4), 464-472. Disponible en: http://dx.doi. org/10.1016/j.giq.2015.09.004.

Mergel, I. y Bretschneider, S. (2013). A Three-Stage Adoption Process for Social Media Use in Government. Public Administration Review, 73 (3), 390-400. Disponible en: http:// dx.doi.org/10.1111/puar.12021.

Mergel, I. y Desouza, K. (2013). Implementing open innovation in the public sector: The case of Challenge.gov. Public Administration Review, 73 (6), 882-890. Disponible en: http:// dx.doi.org/10.1111/puar.12141.

Noveck, B. S. (2009). Wiki Government. How Technology Can Make Government Better, Democracy Stronger, and Citizens More Powerful. Washington D.C.: Brookings Institution Press.

- (2015). Smart Citizens, Smarter State. Cambridge, MA: Hardvard University Press. Disponible en: http://dx.doi.org/10.4159/9780674915435.

Ongaro, E. (2008). Introduction: the reform of public management in France, Greece, Italy, Portugal and Spain. International Journal of Public Sector Management, 21 (2), 101-117. Disponible en: http://dx.doi.org/10.1108/09513550810855618.

Osborne, S. (2006). The New Public Governance? Public Management Review, 8 (3), $337-$ 387. Disponible en: http://dx.doi.org/10.1080/14719030600853022.

Osborne, D., Gaebler, T. (1992). Reinventing Government. How the Entrepreneurial Spirit is Transforming the Public Sector. New York: Addison-Wesley Publishing Company, Inc.

Pardo, T. A. y Gil-García, J. R. y Luna-Reyes, L. F. (2010). Collaborative Governance and Cross-Boundary Information Sharing: Envisioning a Networked and IT-Enabled Public Administration. En R. O'Leary, D. Van Slyke y S. Kim (eds). The Future of Public Administration, Public Management and Public Service around the World: The Minnowbrook Perspective (pp. 129-140). Washington, DC: Georgetown University Press.

Pardo, T. A., Nam, T. y Burke, G. B. (2012). E-Government Interoperability: Interaction of Policy, Management, and Technology Dimensions. Social Science Computer Review, 30 (1), 7-23. Disponible en: http://dx.doi.org/10.1177/0894439310392184.

Parrado, S. (2015). El análisis de la gestión pública. Valencia: Tirant lo Blanch.

Peters, B. G. y Pierre, J. (1998). Governance without Government? Rethinking Public Administration. Journal of Public Administration Research and Theory, 8 (2), 223-243. Disponible en: http://dx.doi.org/10.1093/oxfordjournals.jpart.a024379.

Picazo-Vela, S., Gutiérrez-Martínez, I. y Luna-Reyes, L. F. (2012). Understanding risks, benefits, and strategic alternatives of social media applications in the public sector. Govern- 
ment Information Quarterly, 29, 504-511. Disponible en: http://dx.doi.org/10.1016/j. giq.2012.07.002.

Pollitt, C. (2001). Convergence: the Useful Myth? Public Administration, 79 (4), 933-947. Disponible en: http://dx.doi.org/10.1111/1467-9299.00287.

(2002). The New Public Management in International Perspective. An Analysis of Impacts and Effects. En E. Ferlie, K. McLaughlin y S. B. Osborne (eds.). The New Public Management. Current Trends and Future Prospects (pp. 274-292). London: Routledge.

- (2003). Joined-up Government: A Survey. Political Studies Review, 1 (1), 34-49. Disponible en: http://dx.doi.org/10.1111/1478-9299.00004.

Pollitt, C. y Bouckaert, G. (2011). Public management reform: A comparative analysis: new public management, governance, and the neo-Weberian state. $3^{\text {a }}$ ed. Oxford: Oxford University Press.

Provan, K. G. y Kenis, P. (2008). Modes of network governance: Structure, management, and effectiveness. Journal of Public Administration Research and Theory, 18 (2), 229-252. Disponible en: http://dx.doi.org/10.1093/jopart/mum015.

Ramió, C. (2001). Los problemas de la implantación de la nueva gestión pública en las Administraciones públicas latinas: modelo de Estado y cultura institucional. Reforma y Democracia, 21.

Rhodes, R. A. W. (1996). The New Governance: Governing without Government. Political Studies, 44, 652-667. Disponible en: http://dx.doi.org/10.1111/j.1467-9248.1996.tb01747.x.

- (2007). Understanding Governance: Ten Years On. Organization Studies, 28 (8), 1243 1264. Disponible en: http://dx.doi.org/10.1177/0170840607076586.

Robichau, R. W. (2011). The Mosaic of Governance: Creating a Picture with Definitions, Theories, and Debates. Policy Studies Journal, 39 (s. 1), 113-131. Disponible en: http:// dx.doi.org/10.1111/j.1541-0072.2010.00389_8.x.

Scholl, J. (2014). The EGOV Research Community: An Update on Where We Stand. Ponencia presentada en el 13th International EGOV Conference, Dublín, Irlanda, 1-3 de septiembre. Disponible en: http://dx.doi.org/10.1007/978-3-662-44426-9_1.

Scholl, H. J. y Scholl, M. C. (2014). Smart Governance: A Roadmap for Research and Practice. iConference 2014 Proceedings: Breaking Down Walls. Culture - Context - Computing, 1, 163-176.

Sørensen, E. y Torfing, J. (2012). Collaborative innovation in the public sector. The Innovation Journal: The Public Sector Innovation Journal, 17 (1), 1-14.

Sotiropoulos, D. (2015). Southern European Governments and Public Bureaucracies in the Context of Economic Crisis. European Journal of Social Security, 17 (2), 226-245.

Subirats, J. (2012). ¿Qué democracia y qué Administración para la nueva época? Internet y la gestión pública. Ekonomiaz, 80 (2), 70-91.

Turnbull, N. (2016) Interpreting Governance, High Politics, and Public Policy. Abingdon, Oxon: Routledge.

Van Bueren, E. M., Klijn, E. H. y Koppenjan, J. F. M. (2003). Dealing with Wicked Problems in Networks: Analyzing an Environmental Debate from a Network Perspective. Journal of Public Administration Research and Theory, 13 (2), 193. Disponible en: http://dx.doi. org/10.1093/jopart/mug017. 
Villodre, J. (2016). En la senda de la gobernanza inteligente. Un estudio de la innovación colaborativa en la gestión pública desde el análisis de redes sociales. Tesis de fin de grado. Departamento de Ciencia Política y Relaciones Internacionales. Universidad Autónoma de Madrid.

White House (2009). Memorandum for the Heads of Executive Departments and Agencies, Re: Transparency and Open Government. 21 de enero. Disponible en: https://www. whitehouse.gov/the_press_office/TransparencyandOpenGovernment.

- (2010). Guidance on the Use of Challenges and Prizes to Promote Open Government. 8 de marzo. Disponible en: https://www.whitehouse.gov/sites/default/files/omb/assets/ memoranda_2010/m10-11.pdf.

- (2012). Memorandum for the National Science and Technology Council Committee on Technology: Open Innovator's Toolkit. 8 de febrero. Disponible en: https://www. whitehouse.gov/sites/default/files/microsites/ostp/openinnovatortoolkit_nstcmemo.pdf.

Yildiz, M. (2007). E-government Research. Reviewing the Literature, Limitations, and Ways Forward. Government Information Quarterly, 24 (3), 646-665. Disponible en: http:// dx.doi.org/10.1016/j.giq.2007.01.002. 
